\title{
Shear Viscosity from AdS Born-Infeld Black Holes
}

\author{
Rong-Gen Cai ${ }^{a, 1}$, Ya-Wen Sun ${ }^{a, b}, 2$, \\ a Institute of Theoretical Physics, \\ Chinese Academy of Sciences P.O.Box 2735, Beijing 100190, China \\ ${ }^{b}$ Graduate University of Chinese Academy of Sciences, \\ YuQuan Road 19A, Beijing 100049, China
}

\begin{abstract}
We calculate the shear viscosity in the frame of AdS/CFT correspondence for the field theory with a gravity dual of Einstein-Born-Infeld gravity. We find that the ratio of $\eta / s$ is still the conjectured universal value $1 / 4 \pi$ at least up to the first order of the Born-Infeld parameter $1 / b^{2}$.
\end{abstract}

\footnotetext{
${ }^{1}$ Email: cairg@itp.ac.cn

2Email: sunyw@itp.ac.cn
} 


\section{Introduction}

The AdS/CFT correspondence [1, 2, 3, 4] has been a useful way to calculate dynamical quantities of strongly coupled gauge theories. A famous example is the discovery of the universality of the ratio of the shear viscosity $\eta$ to the entropy density $s$, which is equal to $1 / 4 \pi$ in all theories in the regimes described by gravity duals [5, 6, 7, 8, 9]. This ratio is also conjectured to be a universal lower bound (the KSS bound) for all materials [6]. All known materials in nature by now satisfy this bound. In [10, 11, 12, 13], the authors also calculated the shear viscosity of gauge theories with chemical potentials turned on by studying R-charged black holes. With the presence of nonzero chemical potentials the ratio of shear viscosity to entropy density is still $1 / 4 \pi$. Also the stringy corrections to the ratio were calculated in [14, 15, 16, 17, 18, 19] where the corrections to the value $1 / 4 \pi$ are found to be positive and satisfy the lower bound. More discussions on this KSS bound can be found in [22, 23, 24, 25, 26, 27, 28, 29, 30].

On the other hand, in [20, 21] the authors considered $R^{2}$ corrections in the gravity side and found that the modification of the ratio of shear viscosity over entropy density to the conjectured bound is negative, which means that the lower bound could be violated in that case. The higher derivative gravity corrections they considered can be seen as generated from stringy corrections given the vastness of the string landscape. They gave a new lower bound, $4 / 25 \pi$, based on the causal condition. However, the physical implication of this violation of the bound is still not very clear yet.

This motivates us to consider whether higher derivative corrections to the gauge matter fields on the gravity side also affect the value of shear viscosity when chemical potentials are turned on. In [10, 11, 12, 13], the ratio of $\eta / s$ for the case of nonzero chemical potential was calculated through Einstein-Maxwell theory on the gravity side. As an example of nonlinear electrodynamics, we consider Einstein-Born-Infeld theory with a negative cosmological constant. This theory can be viewed as a nonlinear extension on the gauge fields in Einstein-Maxwell theory.

In this paper we will calculate the shear viscosity of gauge theories with the gravity dual of Einstein-Born-Infeld theory. The background we need is just the AdS Born-Infeld black hole solution [32, 33]. Calculate the shear viscosity in this background and we can see if the higher derivative corrections to the matter fields which are coupled to gravity will also affect the ratio. And the answer we get is that the ratio is not affected by this higher derivative correction to the gauge fields at least at the first non-trivial order. This fact along with the modification of the ratio in higher derivative gravity theories may imply that the ratio may be mainly determined by the form of the action of gravity in the dual gravity description. Here 
the action should be viewed as the effective action which includes the contribution from stringy corrections. If we do not consider the stringy corrections, the effective action for gravity is just the Einstein-Hilbert gravity term. In the case of Einstein-Hilbert gravity, this has been verified in various cases. However, in Gauss-Bonnet gravity the ratio of $\eta / s$ has a correction when gauge fields are coupled to gravity [42].

In this paper, we first present some basic properties of AdS Born-Infeld black holes in Sec. 2. We calculate the shear viscosity of gauge theories with the gravity dual of Einstein-Born-Infeld theory to the first order of the Born-Infeld parameter $1 / b^{2}$ through Kubo-formula in Sec. 3. Sec. 4 is devoted to conclusions and discussions.

\section{AdS Born-Infeld black holes}

In this section we give some basic properties of the Born-Infeld black hole solution in the presence of a negative cosmological constant in five dimensions. The action can be written as

$$
S=\frac{1}{16 \pi G} \int d^{5} x \sqrt{-g}(R-2 \Lambda+L(F)),
$$

where $L(F)=4 b^{2}\left(1-\sqrt{1+\frac{F_{\mu \nu} F^{\mu \nu}}{2 b^{2}}}\right)$. The constant $b$ here is the Born-Infeld parameter and has the dimension of mass. In the limit of $b \rightarrow \infty, L(F)$ reduces to the Maxwell form. Thus if we expand $L(F)$ in a series of $1 / b^{2}$, we will find that the $1 / b^{2}$ corrections to the Maxwell action just correspond to the higher derivative corrections of the gauge fields.

We can write out the equations of motion explicitly as

$$
R_{\mu \nu}-\frac{1}{2} g_{\mu \nu} R+\Lambda g_{\mu \nu}-\frac{1}{2} g_{\mu \nu} L(F)-\frac{2 F_{\mu \lambda} F_{\nu}{ }^{\lambda}}{\sqrt{1+\frac{F^{2}}{2 b^{2}}}}=0
$$

and

$$
\nabla_{\mu}\left(\frac{F^{\mu \nu}}{\sqrt{1+\frac{F^{2}}{2 b^{2}}}}\right)=0
$$

Since we want to calculate the shear viscosity of the corresponding field theory living in $R^{1,3}$, we need a black hole solution with a Ricci-flat horizon. The AdS Born-Infeld black hole solution with a Ricci-flat horizon can be written as 33

$$
d s^{2}=-V(r) d t^{2}+\frac{1}{V(r)} d r^{2}+r^{2}\left(d \vec{x}^{2}\right)
$$

where

$$
V(r)=-\frac{m}{r^{2}}+\left(\frac{b^{2}}{3}+\frac{1}{l^{2}}\right) r^{2}-\frac{b}{3 r} \sqrt{b^{2} r^{6}+3 q^{2}}+\frac{3 q^{2}}{2 r^{4}}{ }_{2} F_{1}\left[\frac{1}{3}, \frac{1}{2}, \frac{4}{3},-\frac{3 q^{2}}{b^{2} r^{6}}\right],
$$


and the only nonzero component of $F_{\mu \nu}$ is

$$
F^{r t}=\frac{\sqrt{3} b q}{\sqrt{b^{2} r^{6}+3 q^{2}}} .
$$

Here $q$ is an integration constant which is related to the electric charge of the black hole and $l$ is the AdS radius through $\Lambda=-6 / l^{2}$. When $b^{2}$ approaches to infinity, this solution becomes the AdS Reissner-Nordström black hole solution.

We can get the position of the outer horizon by solving $V\left(r_{+}\right)=0$. For future convenience we define $u=r_{+}^{2} / r^{2}$ and rescale $\vec{x}$ to the new coordinate system

$$
d s^{2}=-V(u) d t^{2}+\frac{r_{+}^{2}}{4 u^{3} V(u)} d u^{2}+\frac{r_{+}^{2}}{u l^{2}} d \vec{x}^{2} .
$$

Now $V(u)$ becomes

$$
V(u)=-\frac{m u}{r_{+}^{2}}+\left(\frac{b^{2}}{3}+\frac{1}{l^{2}}\right) \frac{r_{+}^{2}}{u}-\frac{b}{3 r_{+}} \sqrt{\frac{b^{2} r_{+}^{6}}{u^{2}}+3 q^{2} u}+\frac{3 q^{2} u^{2}}{2 r_{+}^{4}}{ }_{2} F_{1}\left[\frac{1}{3}, \frac{1}{2}, \frac{4}{3},-\frac{3 q^{2} u^{3}}{b^{2} r_{+}^{6}}\right],
$$

and the horizon corresponds to $u=1$. Then the mass parameter $m$ can be expressed by $r_{+}$as

$$
m=\left(\frac{b^{2}}{3}+\frac{1}{l^{2}}\right) r_{+}^{4}-\frac{b r_{+}}{3} \sqrt{b^{2} r_{+}^{6}+3 q^{2}}+\frac{3 q^{2}}{2 r_{+}^{2}}{ }_{2} F_{1}\left[\frac{1}{3}, \frac{1}{2}, \frac{4}{3},-\frac{3 q^{2}}{b^{2} r_{+}^{6}}\right] .
$$

The thermodynamic properties of this black hole has been discussed in [32, 33, 34, 35, 36, 37]. Here we only give the entropy density of this black hole solution for future use

$$
s=\frac{1}{4 G} \frac{r_{+}^{3}}{l^{3}} .
$$

\section{Shear viscosity from AdS Born-Infeld black holes}

In this section we calculate the shear viscosity of the field theory dual to the black hole background (44) through the Kubo-formula [22, 38]:

$$
\eta=\lim _{\omega \rightarrow 0} \frac{1}{2 \omega i}\left(G_{x y, x y}^{A}(\omega, 0)-G_{x y, x y}^{R}(\omega, 0)\right),
$$

where $\eta$ is the shear viscosity, and the retarded Green's function is defined by

$$
G_{\mu \nu, \lambda \rho}^{R}(k)=-i \int d^{4} x e^{-i k \cdot x} \theta(t)\left\langle\left[T_{\mu \nu}(x), T_{\lambda \rho}(0)\right]\right\rangle .
$$

The advanced Green's function can be related to the retarded Green's function by $G_{\mu \nu, \lambda \rho}^{A}(k)=$ $G_{\mu \nu, \lambda \rho}^{R}(k)^{*}$. We compute the retarded Green's function by making a small perturbation of 
graviton. Here we choose spatial coordinates so that the momentum of the perturbation points along the $z$-axis. Then the perturbations can be written as $h_{\mu \nu}=h_{\mu \nu}(t, z, r)$. In this basis there are three groups of gravity perturbations, each of which is decoupled from others: the scalar, vector and tensor perturbations [31]. Here we use the simplest one, the tensor perturbation $h_{x y}$. We use $\phi$ to denote this perturbation $\phi=h_{y}^{x}$ and write $\phi$ in a basis as $\phi(t, u, z)=\phi(u) e^{-i \omega t+i p z}$.

We can get the equation of motion of this $\phi(u)$ by perturbing both sides of the equation of motion (2) to the first order of $\phi(u)$

$$
\phi^{\prime \prime}(u)+A \phi^{\prime}(u)+B \phi(u)=0 .
$$

Here to avoid complication we only calculate the shear viscosity up to the first order in the parameter $1 / b^{2}$, and in this approximation we have

$$
A=A_{0}+A_{1}
$$

where $A_{0}$ denotes the part of the coefficient $A$ in the limit $b^{2} \rightarrow \infty$,

$$
A_{0}=\frac{l^{2} q^{2}(1-2 u) u^{2}+r_{+}^{6}\left(1+u^{2}\right)}{u(u-1)\left(r_{+}^{6}(1+u)-l^{2} q^{2} u^{2}\right)},
$$

while $A_{1}$ denotes the part of the first order correction of $1 / b^{2}$ :

$$
A_{1}=\frac{3 l^{2} q^{4} u\left(2 r_{+}^{6}(1+u)^{2}\left(1+2 u^{2}\right)-l^{2} q^{2} u^{3}\left(1+2 u+3 u^{2}\right)\right)}{16 b^{2} r_{+}^{6}\left(l^{2} q^{2} u^{2}-r_{+}^{6}(1+u)\right)^{2}} .
$$

Also $B$ can be written as the sum of two parts,

$$
\begin{aligned}
B & =B_{0}+B_{1} \\
& =\frac{r_{+}^{6}\left(\bar{p}^{2}(u-1)\left(r_{+}^{6}(1+u)-l^{2} q^{2} u^{2}\right)+r_{+}^{6} \bar{w}^{2}\right)}{u(u-1)^{2}\left(r_{+}^{6}(1+u)-l^{2} q^{2} u^{2}\right)^{2}} \\
& +\frac{3 l^{2} q^{4} u\left(1+u+u^{2}+u^{3}\right)\left(\bar{p}^{2}(u-1)\left(-r_{+}^{6}(1+u)+l^{2} q^{2} u^{2}\right)-2 r_{+}^{6} \bar{w}^{2}\right)}{16 b^{2}(u-1)^{2}\left(r_{+}^{6}(1+u)-l^{2} q^{2} u^{2}\right)^{3}} .
\end{aligned}
$$

Here $\bar{w}=l^{2} \omega / 2 r_{+}$and $\bar{p}=l^{2} p / 2 r_{+} . \quad A_{1}$ and $B_{1}$ manifest the contribution of the higher derivative corrections to the gauge field on the gravity side. To solve for $\phi(u)$, we write

$$
\phi(u)=(1-u)^{-i \beta \bar{w}} F(u)
$$

to decide the boundary condition near the horizon, where $\beta$ is a constant to be fixed. Substituting (18) into (13) and solving it near the horizon $u=1$, we get

$$
\beta=\beta_{0}+\beta_{1}=\frac{r_{+}^{6}}{2 r_{+}^{6}-l^{2} q^{2}}-\frac{3 l^{2} q^{4}}{4 b^{2}\left(2 r_{+}^{6}-l^{2} q^{2}\right)^{2}},
$$


by pure incoming wave boundary condition near $u=1$. Here $\beta_{1}$ is the contribution of the higher derivative correction. Next we move on to solve $\phi(u)$ in the whole spacetime. Because we know from (11) that we only need the low frequency behavior of $\phi(u)$ to calculate the shear viscosity, we can expand $F(u)$ in a power series of $\bar{w}$ and $\bar{p}$

$$
F(u)=1+i \beta_{0} \bar{w} F_{0}(u)+i \beta_{1} \bar{w} F_{1}(u)+O\left(\bar{w}^{2}\right)+O\left(\bar{p}^{2}\right)
$$

In this expansion, $F_{1}$ represents the contribution from the higher derivative correction of the gauge field. The equations of motion of $F_{0}(u)$ and $F_{1}(u)$ can be derived at the first order of $\bar{w}$ separately,

$$
F_{0}^{\prime \prime}(u)+A_{0} F_{0}^{\prime}(u)+\frac{1}{(1-u)^{2}}+\frac{A_{0}}{1-u}=0
$$

and

$$
F_{1}^{\prime \prime}(u)+A_{0} F_{1}^{\prime}(u)+\frac{\beta_{0}}{\beta_{1}} A_{1} F_{0}^{\prime}(u)+\frac{\beta_{0} A_{1}}{\beta_{1}(1-u)}+\frac{1}{(1-u)^{2}}+\frac{A_{0}}{1-u}=0 .
$$

The solutions of these two linear differential equations can be uniquely decided with the boundary condition $\left.F_{0}(u)\right|_{u=0}=\left.F_{1}(u)\right|_{u=0}=0$ and the constraint that both $F_{0}(u)$ and $F_{1}(u)$ should be regular at the horizon $u=1$. The solutions are

$$
F_{0}(u)=\frac{1}{2}\left(\ln \frac{r_{+}^{6}(1+u)-l^{2} q^{2} u^{2}}{r_{+}^{6}}-\frac{3 r_{+}^{3}}{\sqrt{4 l^{2} q^{2}+r_{+}^{6}}} \ln \frac{u \sqrt{4 l^{2} q^{2}+r_{+}^{6}}+r_{+}^{3}(2+u)}{-u \sqrt{4 l^{2} q^{2}+r_{+}^{6}}+r_{+}^{3}(2+u)}\right),
$$

and

$$
\begin{aligned}
F_{1}(u) & =C_{0}(u)+C_{1} \ln \frac{\left(r_{+}^{3} \sqrt{4 l^{2} q^{2}+r_{+}^{6}}+r_{+}^{6}-2 l^{2} q^{2} u\right)\left(\sqrt{4 l^{2} q^{2}+r_{+}^{6}}-r_{+}^{3}\right)}{\left(r_{+}^{3} \sqrt{4 l^{2} q^{2}+r_{+}^{6}}-r_{+}^{6}+2 l^{2} q^{2} u\right)\left(\sqrt{4 l^{2} q^{2}+r_{+}^{6}}+r_{+}^{3}\right)} \\
& +C_{2} \ln \frac{r_{+}^{6}(1+u)-l^{2} q^{2} u^{2}}{r_{+}^{6}}
\end{aligned}
$$

where $C_{0}(u)$ is a function of $u$,

$$
\begin{aligned}
C_{0}(u)=- & \frac{\left(l^{2} q^{2}-2 r_{+}^{6}\right) u}{8 l^{6} q^{6}\left(4 l^{2} q^{2}+r_{+}^{6}\right)\left(l^{2} q^{2} u^{2}-r_{+}^{6}(1+u)\right)} \times \\
& \left(12 r_{+}^{24}(1+u)-6 l^{2} q^{2} r_{+}^{18}\left(-9-10 u+u^{2}\right)+4 l^{8} q^{8} u\left(1+4 u+u^{2}\right)+\right. \\
& \left.l^{4} q^{4} r_{+}^{12}\left(30+51 u-29 u^{2}-2 u^{3}\right)-l^{6} q^{6} r_{+}^{6}\left(6+2 u+16 u^{2}+7 u^{3}\right)\right),
\end{aligned}
$$

$C_{1}$ and $C_{2}$ are two constants,

$$
\begin{aligned}
C_{1} & =\frac{3\left(10 l^{10} q^{10} r_{+}^{3}+25 l^{8} q^{8} r_{+}^{9}+12 l^{6} q^{6} r_{+}^{15}-45 l^{4} q^{4} r_{+}^{21}-28 l^{2} q^{2} r_{+}^{27}-4 r_{+}^{33}\right)}{8 l^{8} q^{8}\left(4 l^{2} q^{2}+r_{+}^{6}\right)^{3 / 2}} \\
C_{2} & =\frac{l^{8} q^{8}+6 l^{6} q^{6} r_{+}^{6}+9 l^{4} q^{4} r_{+}^{12}-12 l^{2} q^{2} r_{+}^{18}-12 r_{+}^{24}}{8 l^{8} q^{8}}
\end{aligned}
$$


Now we want to get the on-shell action for the perturbation. The on-shell action for $\phi(u)$ is a sum of two parts: one is from the bulk action $S_{\text {bulk }}$ and the other from the Gibbons-Hawking boundary term $S_{G H}$. We first expand

$$
\phi(x, u)=\int \frac{d^{4} k}{(2 \pi)^{4}} e^{i k x} f(k) \phi_{k}(u) .
$$

The bulk action for this perturbation then is

$$
S_{\text {bulk }}=\frac{1}{16 \pi G} \int \frac{d^{4} k}{(2 \pi)^{4}} f(k) f(-k) \int_{1}^{0} d u\left(K_{1} \phi_{k}^{\prime \prime} \phi_{-k}+K_{2} \phi_{k}^{\prime} \phi_{-k}^{\prime}+K_{3} \phi_{k}^{\prime} \phi_{-k}+K_{4} \phi_{k} \phi_{-k}\right)
$$

where $K_{1}=2 \sqrt{-g} g^{u u}$ and $K_{2}=\frac{3}{2} \sqrt{-g} g^{u u}$. The $K_{3}$ and $K_{4}$ terms are not relevant to our aim, so we do not explicitly write them here. The Gibbons-Hawking boundary term is

$$
S_{G H}=\frac{1}{8 \pi} \int_{\partial M} d^{4} x \sqrt{-h} K
$$

Substituting the solution of $\phi(u)$ into (30) and we reach

$$
S_{G H}=\frac{1}{16 \pi} \int \frac{d^{4} k}{(2 \pi)^{4}} f(k) f(-k)\left(K_{5} \phi_{k} \phi_{-k}+K_{6} \phi_{k}^{\prime} \phi_{-k}\right)
$$

where $K_{6}=-2 \sqrt{-g} g$ au $K_{5}$ only contributes to the Green's function a real part thus not relevant to the following calculations.

By applying the equation of motion (13), the bulk action becomes two surface terms

$$
S_{\text {bulk }}=\frac{1}{16 \pi G} \int \frac{d^{4} k}{(2 \pi)^{4}} f(k) f(-k)\left(\int_{1}^{0} d u[E . O . M] \phi_{-k}+\left.\left(\frac{K_{3}-K_{1}^{\prime}}{2} \phi_{k} \phi_{-k}+K_{2} \phi_{k}^{\prime} \phi_{-k}\right)\right|_{1} ^{0}\right) .
$$

The Gibbons-Hawking term is itself a boundary contribution, so the total action can be written as

$$
S=\left.\int \frac{d^{4} k}{(2 \pi)^{4}} f(k) f(-k) F(k, u)\right|_{0} ^{1}
$$

where $F(k, u)$ can be expressed by the constants $K_{i}$. The retarded Green's function can be calculated in the way

$$
G_{x y, x y}^{R}(k)=-2 F(k, u=0) .
$$

We substitute the solution into (33) and can obtain

$$
F(k, u=0)=\left.\frac{1}{32 \pi G} \sqrt{-g} g^{u u} \phi_{k}^{\prime} \phi_{k}^{*}\right|_{u=0}=\frac{1}{16 \pi G} i \bar{w} \frac{r_{+}^{4}}{l^{5}}=\frac{1}{16 \pi G} i \omega \frac{r_{+}^{3}}{2 l^{3}} .
$$

Substituting this into (34) and (11), we have

$$
\eta=\lim _{\omega \rightarrow 0} \frac{F-F^{*}}{w i}=\frac{1}{16 \pi G} \frac{r_{+}^{3}}{l^{3}} .
$$


Comparing this with $s=\frac{1}{4 G} \frac{r_{+}^{3}}{l^{3}}$, we finally reach $\eta / s=1 / 4 \pi$.

Here we only performed the calculation to the first order of the parameter $1 / b^{2}$. That is to say, we considered the effect on the ratio of $\eta / s$ of the electromagnetic field corrected term $\left(F_{\mu \nu} F^{\mu \nu}\right)^{2}$. Here we further show that at this order the term, $F_{\mu \nu} F^{\nu \rho} F_{\rho \sigma} F^{\sigma \mu}$, has also no contribution to the shear viscosity. In this case, the action we are considering can be written as

$$
S=\frac{1}{16 \pi G} \int d^{5} x \sqrt{-g}(R-2 \Lambda+I(F))
$$

where

$$
I(F)=-F_{\mu \nu} F^{\mu \nu}+\frac{\left(F_{\mu \nu} F^{\mu \nu}\right)^{2}}{8 b^{2}}+c F_{\mu \nu} F^{\nu \rho} F_{\rho \sigma} F^{\sigma \mu}
$$

Here $b$ and $c$ can be arbitrary constants which represent the effects of the higher derivative corrections of the gauge fields. The term proportional to $1 / b^{2}$ is the one appearing as the first order correction of the Born-Infeld action. As the case of the AdS Born-Infeld black holes, we consider a Ricci flat black hole solution with electric charge in the action (37). In this case, the only nonvanishing component of electromagnetic field is $F_{t r}$. The term $\left(F_{\mu \nu} F^{\mu \nu}\right)^{2}$ is then just twice of $F_{\mu \nu} F^{\nu \rho} F_{\rho \sigma} F^{\sigma \mu}$. Thus the results concerning the corrected term only depend on the combination of the two coefficients by $1 / 4 b^{2}+c$ [40]. That is, at this order, we can obtain the black hole metric and electric field for the action (37) by replacing the coefficient $1 / b^{2}$ in the Born-Infeld black hole metric and electric field with the coefficient $1 / b^{2}+4 c$. In the above, we have already shown that the coefficient $1 / b^{2}$ does not explicitly appear in the shear viscosity. We then conclude that the term $F_{\mu \nu} F^{\nu \rho} F_{\rho \sigma} F^{\sigma \mu}$ also will not change the ratio of the shear viscosity to the entropy density.

\section{Conclusions and Discussions}

In this paper we calculated the ratio of shear viscosity to entropy density in the background of AdS Born-Infeld black holes through AdS/CFT correspondence to the first order of the BornInfeld parameter $1 / b^{2}$. Motivated by [20] we find that though the higher derivative corrections to the gravity term make the universal bound be modified, the higher derivative corrections to the gauge fields have no change on the value of the $\eta / s$ ratio at least at the first non-trivial order.

This result may give us some hint on in what sense the lower bound is universal. We learn that when no gravity corrections are considered, the ratio of $\eta / s$ is the same for various gravity backgrounds [7] and for gauge theories with nonzero chemical potentials. When gravity corrections are added, the ratio changes and even the lower bound could be violated, but higher 
derivative corrections to the gauge fields on the gravity side do not change the value of $\eta / s$. The difference between these two corrections is that with the gravity correction the effective action of gravity part is changed while in the latter case the effective action of gravity part is still the Einstein-Hilbert form though matter fields are coupled to gravity in various ways. This may imply that the universality of the ratio $1 / 4 \pi$ is just the universality among gauge field theories which have Einstein-Hilbert gravity as their gravity duals which can couple to arbitrary matter fields through various proper ways. In other cases the ratio should be mainly determined by the effective gravity part of the dual gravity description [41]. The result of [17, 39] might be viewed as evidence to support this idea, where the author found some evidence of universality of shear viscosity at finite t'Hooft coupling. In a recent paper [42, it has been observed that in the case of Gauss-Bonnet gravity, the existence of chemical potentials changes the ratio, too.

\section{Acknowledgements}

This work was supported in part by a grant from Chinese Academy of Sciences, grants from NSFC with No. 10325525 and No. 90403029.

\section{References}

[1] J. M. Maldacena, Adv. Theor. Math. Phys. 2, 231 (1998) [Int. J. Theor. Phys. 38, 1113 (1999)] arXiv:hep-th/9711200].

[2] S. S. Gubser, I. R. Klebanov and A. M. Polyakov, Phys. Lett. B 428, 105 (1998) |arXiv:hep-th/9802109|.

[3] E. Witten, Adv. Theor. Math. Phys. 2, 253 (1998) [arXiv:hep-th/9802150].

[4] O. Aharony, S. S. Gubser, J. M. Maldacena, H. Ooguri and Y. Oz, Phys. Rept. 323, 183 (2000) arXiv:hep-th/9905111.

[5] G. Policastro, D. T. Son and A. O. Starinets, Phys. Rev. Lett. 87, 081601 (2001) arXiv:hep-th/0104066].

[6] P. Kovtun, D. T. Son and A. O. Starinets, JHEP 0310, 064 (2003) arXiv:hep-th/0309213.

[7] A. Buchel and J. T. Liu, Phys. Rev. Lett. 93, 090602 (2004) arXiv:hep-th/0311175.

[8] P. Kovtun, D. T. Son and A. O. Starinets, Phys. Rev. Lett. 94, 111601 (2005) arXiv:hep-th/0405231].

[9] A. Buchel, Phys. Lett. B 609, 392 (2005) [arXiv:hep-th/0408095].

[10] J. Mas, JHEP 0603, 016 (2006) arXiv:hep-th/0601144.

[11] D. T. Son and A. O. Starinets, JHEP 0603, 052 (2006) arXiv:hep-th/0601157. 
[12] O. Saremi, JHEP 0610, 083 (2006) arXiv:hep-th/0601159.

[13] K. Maeda, M. Natsuume and T. Okamura, Phys. Rev. D 73, 066013 (2006) arXiv:hep-th/0602010].

[14] A. Buchel, J. T. Liu and A. O. Starinets, Nucl. Phys. B 707, 56 (2005) |arXiv:hep-th/0406264].

[15] P. Benincasa and A. Buchel, JHEP 0601, 103 (2006) arXiv:hep-th/0510041.

[16] A. Buchel, arXiv:0801.4421 [hep-th].

[17] A. Buchel, arXiv:0804.3161 [hep-th].

[18] A. Buchel, arXiv:0805.2683 [hep-th].

[19] R. C. Myers, M. F. Paulos and A. Sinha, arXiv:0806.2156 [hep-th].

[20] M. Brigante, H. Liu, R. C. Myers, S. Shenker and S. Yaida, Phys. Rev. D 77, 126006 (2008) arXiv:0712.0805 [hep-th]]; M. Brigante, H. Liu, R. C. Myers, S. Shenker and S. Yaida, Phys. Rev. Lett. 100, 191601 (2008) [arXiv:0802.3318 [hep-th]].

[21] Y. Kats and P. Petrov, arXiv:0712.0743 [hep-th].

[22] G. Policastro, D. T. Son and A. O. Starinets, JHEP 0209, 043 (2002) |arXiv:hep-th/0205052].

[23] G. Policastro, D. T. Son and A. O. Starinets, JHEP 0212, 054 (2002) arXiv:hep-th/0210220.

[24] T. D. Cohen, Phys. Rev. Lett. 99, 021602 (2007) arXiv:hep-th/0702136].

[25] A. Cherman, T. D. Cohen and P. M. Hohler, JHEP 0802, 026 (2008) arXiv:0708.4201 [hep-th]].

[26] J. W. Chen, M. Huang, Y. H. Li, E. Nakano and D. L. Yang, arXiv:0709.3434 [hep-ph].

[27] D. T. Son, Phys. Rev. Lett. 100, 029101 (2008) [arXiv:0709.4651 [hep-th]].

[28] I. Fouxon, G. Betschart and J. D. Bekenstein, Phys. Rev. D 77 (2008) 024016 arXiv:0710.1429 [gr-qc]].

[29] A. Dobado, F. J. Llanes-Estrada and J. M. T. Rincon, arXiv:0804.2601 [hep-ph].

[30] K. Landsteiner and J. Mas, JHEP 0707, 088 (2007) arXiv:0706.0411 [hep-th]].

[31] P. K. Kovtun and A. O. Starinets, Phys. Rev. D 72, 086009 (2005) arXiv:hep-th/0506184.

[32] T. K. Dey, Phys. Lett. B 595, 484 (2004) [arXiv:hep-th/0406169].

[33] R. G. Cai, D. W. Pang and A. Wang, Phys. Rev. D 70, 124034 (2004) |arXiv:hep-th/0410158].

[34] S. Fernando, Phys. Rev. D 74, 104032 (2006) arXiv:hep-th/0608040.

[35] A. Sheykhi and N. Riazi, Phys. Rev. D 75, 024021 (2007) arXiv:hep-th/0610085].

[36] O. Miskovic and R. Olea, Phys. Rev. D 77, 124048 (2008) [arXiv:0802.2081 [hep-th]]. 
[37] Y. S. Myung, Y. W. Kim and Y. J. Park, arXiv:0805.0187 [gr-qc].

[38] D. T. Son and A. O. Starinets, Ann. Rev. Nucl. Part. Sci. 57, 95 (2007) arXiv:0704.0240 [hep-th]].

[39] A. Buchel, R. C. Myers, M. F. Paulos and A. Sinha, arXiv:0808.1837 [hep-th].

[40] D. Anninos and G. Pastras, arXiv:0807.3478 [hep-th].

[41] R. Brustein and A. J. M. Medved, arXiv:0808.3498 [hep-th].

[42] X. H. Ge, Y. Matsuo, F. W. Shu, S. J. Sin and T. Tsukioka, arXiv:0808.2354 [hep-th]. 